Ann. Biol. anim. Bioch. Biophys., I969, 9 (3), 405-4I2.

\title{
INFLUENCE DE PRODUITS SOLUBLES FORMES PAR CHAUFFAGE DU GLUCOSE ET DU GLYCOCOLLE SUR L'HYDROLYSE ENZYMATIQUE DE DIFFERENTES PROTÉINES
}

\author{
M. SUSCHETET \\ Station de Recherches sur les Aliments de l'Homme, \\ Centre de Recherches agronomiques de Dijon \\ Institut national de la Recherche agronomique
}

\section{SOMMAIRE}

En chauffant à $90^{\circ} \mathrm{C}$ pendant I heure ou 6 heures un mélange de glucose et de glycocolle, on obtient un ensemble de produits solubles (prémélanoïdines) dont l'influence a été étudiée sur l'hydrolyse in vitro par la pepsine, la trypsine et la peptidase, de quelques protéines.

A la concentration de roo $\mathrm{mg} / \mathrm{g}$ de protéines, les prémélanoïdines " $\mathrm{I} h 90^{\circ}$ " n'ont pas d'influence sur l'hydrolyse de la caséine, de l'ovalbumine, du blanc d'œuf cru ou cuit et du gluten de blé.

Quant aux prémélanoïdines " $6 \mathrm{~h} 90^{\circ}$ ", alors qu'une dose de $50 \mathrm{mg} / \mathrm{g}$ de protéines n'affecte pas l'hydrolyse de ces protéines, une dose ro fois plus forte entraîne une diminution de l'azote aminé libéré lors de l'hydrolyse de l'ovalbumine et du blanc d'œuf : la protéolyse des autres substrats n'étant pas touchée, il semble logique de rechercher la cause de ce retard à l'hydrolyse dans les caractéristiques propres des protéines de l'œuf.

\section{INTRODUCTION}

Parmi toutes les techniques tendant à transformer les produits alimentaires, une large place doit être faite aux traitements thermiques : ils sont en effet largement employés par les industries alimentaires, soit pour prolonger la durée de conservation de denrées périssables, soit pour modifier les caractéristiques organoleptiques de certains produits. Mais, au cours de ces traitements peuvent apparaître des modifications dans la structure des constituants biochimiques fondamentaux de ces aliments : la réaction de Maillard, qui intervient entre glucides réducteurs et protéines, et dont la première manifestation est un brunissement du milieu sans intervention enzymatique, est une de ces modifications. 
Les problèmes posés par l'existence de cette réaction peuvent être non seulement d'ordre analytique (difficultés de dosages de sucres et d'amino-acides dans un milieu où la réaction de Maillard s'est développée) et d'ordre technologique (les industries des céréales, la malterie, la brasserie... en sont directement tributaires), mais aussi d'ordres physiologique et nutritionnel.

On sait en effet d'une part, que la réaction de Maillard provoque la destruction de la molécule du sucre et celle des acides aminés, en particulier la lysine, et qu'elle est ainsi à l'origine d'un abaissement de la valeur biologique des protéines.

D'autre part, il semble bien que les protéines touchées par cette réaction soient plus difficilement attaquables par les enzymes digestifs.

On peut aussi se demander si certains des nombreux produits de dégradation formés dans cette réaction ne sont pas susceptibles de modifier le déroulement normal des réactions métaboliques chez les êtres vivants.

ADRIAN et al. (1966) ont en effet mis en évidence un phénomène de perturbation de la croissance (ralentissement ou activation suivant les cas) chez le Rat ingérant, en plus de son régime, des doses variables de composés formés par chauffage du mélange d'un sucre et d'un acide aminé.

Des études concernant l'influence de ces composés sur la croissance de la levure (Sheikh, Godon et Petit, r96r) et d'autres types de microorganismes (Jemmali, I965) ont conduit leurs auteurs à rechercher une action directe de ces produits sur certains systèmes enzymatiques (décarboxylase de l'acide pyruvique de la levure).

Dans le même ordre d'idées, on peut se demander si la présence de produits de la réaction de Maillard dans une ration alimentaire ne risque pas d'affecter l'ensemble des mécanismes des actions des enzymes de la digestion. A ce propos, PetrT et AdRIAN (I967) signalent que l'érepsine se montre sensible à la présence de ces produits.

Nous avons donc, pour ces différentes raisons, abordé l'étude de l'influence des produits de la réaction de Maillard sur le déroulement, in vitro, de 1'hydrolyse de quelques substrats protéiques courants.

\section{MATÉRIEL ET MÉTHODES}

Ce travail présente les résultats d'une série d'expériences sur l'hydrolyse in vitro de différents substrats protéiques par des enzymes digestifs (pepsine, trypsine, peptidase), en présence ou en l'absence de prémélanoïdines de glucose et de glycocolle.

Cette dénomination de " prémélanoïdines " est attachée à l'ensemble des produits résultant de la réaction de Maillard solubles dans l'eau. Un stade ultérieur de la dégradation fait apparaître une série de corps insolubles qu'on réunit sous le terme de " mélanoïdines".

Nous n'avons retenu, dans cette étude, que les prémélanoïdines issues d'un mélange de gluc ose et de glycocolle porté à $90^{\circ} \mathrm{C}$.

\section{A. - Conditions d'obtention des prémélanoïdines}

Nous nous sommes inspiré des techniques utilisées dans ce domaine par ADRIAN et al. (Ig66)

Dans un réacteur en verre, constitué d'un tube à essai à col rodé surmonté d'un réfrigérant à eau, on introduit un mélange intime de $3,6 \mathrm{~g}$ de glucose et $\mathrm{I}, 5 \mathrm{~g}$ de glycocolle, ainsi qu'une faible quantité d'eau ( $\mathrm{I} \mathrm{ml}$ ) de façon à obtenir un mélange " pâteux " dont l'hydratation initiale est de 19,5 p. 100 .

Le réacteur est immergé dans un bain thermostaté à température réglable, les produits de la réaction étant alors caractérisés par la durée du chauffage subi et par la température. 


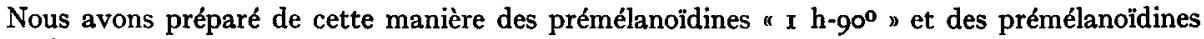
" $6 \mathrm{~h}-90^{\circ}$ ".

Le contenu de chaque réacteur est, en fin de réaction, récupéré par de l'eau distillée : les prémélanoïdines " I $\mathrm{h}-90^{\circ}$ " sont amenées à une concentration $2 \mathrm{M}$ en glucose et glycocolle initiaux, tandis que les prémélanoïdines \& $6 \mathrm{~h}-90^{\circ}$, de consistance semi-liquide, sont amenées à une concentration $\mathrm{M}$ en glucose et glycocolle initiaux.

Dans le processus expérimental où sont engagés ces produits de la réaction de Maillard, i' est plus pratique de les utiliser sous forme sèche : les solutions de récupération " I h-90" " et " $6 \mathrm{~h}-90^{\circ}$ " sont donc lyophilisées et conservées au froid.

\section{B. - Caractéristiques analytiques des prémélanoidines}

Pour caractériser les produits obtenus, nous avons procédé à différents dosages établissant le degré de dégradation subi par le sucre et l'acide aminé engagés initialement :

taux de glucose restant intact après chauffage,

taux de glycocolle,

pouvoir réducteur total des produits obtenus.

Pour la détermination du glycocolle restant, on isole l'acide aminé demeurant dans les prémélanoïdines par une chromatographie monodimensionnelle descendante sur papier, dans un solvant constitué par 4 volumes de butanol, I volume d'acide acétique et 5 volumes d'eau. Après i $6 \mathrm{~h}$ d'élution, le chromatogramme est révélé à la ninhydrine selon STARoN (196r) (réactif : acétone $72 \mathrm{ml}$; cyclohexanol $30 \mathrm{ml}$; ninhydrine $2 \mathrm{~g}$; eau $10 \mathrm{ml}$ ). On procède à un séchage sous hotte pendant $15 \mathrm{mn}$, puis à l'étuve à $65^{\circ}$ pendant $3 \mathrm{mn}$, et enfin à l'air pendant $\mathrm{I}$ heure. Ces taches révélées sont alors éluées par de l'éthanol à $5 \circ \mathrm{p}$. roo et leur densité optique, lue à $570 \mathrm{~m} \mu$, est rapportée à celle d'un étalon de glycocolle. La précision de cette méthode est de l'ordre de $8 \mathrm{p}$. Ioo.

La méthode employée pour le dosage du glucose résiduel est la méthode enzymatique à la glucose-oxydase (HugETT et Nixon, 1957) modifiée par GuIDOTTI $t$ t al. (1961), dont la précision est de l'ordre de $2 \mathrm{p}$. тоo. Pour la détermination du pouvoir réducteur total, on utilise la méthode de SchafFer et Hartmann (1920), et les résultats sont exprimés en glucose.

Les résultats de ces analyses sont présentés dans le tableau I.

\section{C. - Origine et caractéristiques des substrats utilisés}

Six substrats protéiques ont été utilisés :

Teneur en protéines

Caséine lactique (GAllia) $\ldots \ldots \ldots \ldots \ldots \ldots \ldots$

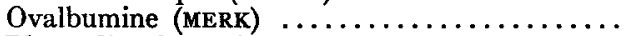

Blanc d'œuf cru (ECH. LABo.) ............

Blanc d'œuf cuit (ЕсH. LABO.) ............

Gluten (N. B. C.) ...................

Gluten (ЕСН. LABo.) ...............
73,5 p. $100(\mathrm{~N} \times 6,38)$

95,3 p. $100(\mathrm{~N} \times 6,25)$

75,0 p. $100(\mathrm{~N} \times 6,25)$

75,0 p. $100(\mathrm{~N} \times 6,25)$

$7 \mathrm{I}, 6$ p. I00 $(\mathrm{N} \times 5,75)$

82,2 p. $100(\mathrm{~N} \times 5,75)$

Le gluten préparé au laboratoire a été extrait par lixiviation à l'eau à partir d'une farine Ruban $B l e u$, puis lyophilisé sur plateaux et pulvérisé au moulin de laboratoire Bühler.

Le blanc d'ouf cru a été obtenu à partir d'œufs frais ; après élimination de la chalaze par une filtration sur gaze, le blanc a été lyophilisé, puis pulvérisé.

Le blanc d'œuf cuit a été séparé après cuisson des œufs au bain-marie à $100^{\circ}$ pendant $15 \mathrm{mn}$, puis lyophilisé et pulvérisé.

\section{D. - Techniques d'hydrolyse}

On introduit dans un matras à fond plat $\mathrm{I} g$ de protéine et une quantité de prémélanoïdines, variable suivant les essais. Le matras est immergé dans un bain-marie à $40^{\circ} \mathrm{C}$, l'agitation étant assurée par des agitateurs magnétiques.

On verse $25 \mathrm{ml}$ d'une solution $0, \mathrm{I} \mathrm{N}$ en acide chlorhydrique contenant la pepsine à la concen. tration de $2 \mathrm{mg} / \mathrm{ml}$ (pepsine $\mathrm{I} / \mathrm{r} 0$ 000 Difco).

Après $30 \mathrm{mn}$ de cette attaque pepsique, le contenu du matras est neutralisé par $12,5 \mathrm{ml}$ de soude $0,2 \mathrm{~N}$ et un prélèvement de $5 \mathrm{ml}$ de l'hydrolysat pepsique est effectué. On introduit alors dans le matras $15 \mathrm{ml}$ d'une solution o, I $\mathrm{M}$ en phosphate disodique à $2,5 \mathrm{mg} / \mathrm{ml}$ de trypsine (trypsine $\mathbf{r} / 25^{\circ} \mathrm{Difco}$ ) et à $4 \mathrm{mg} / \mathrm{ml}$ de peptidase (Peptidase " Porcine Intestine $n-\mathrm{C}$ grade Calbiochem). 
Des prélèvements de $5 \mathrm{ml}$ sont effectués aux temps $5 \mathrm{mn}, 30 \mathrm{mn}$, $\mathrm{x}$ heure et 3 heures de l'hydrolyse trypsine-peptidase.

Chaque prélèvement est immédiatement soumis à une ébullition de $15 \mathrm{mn}$ sous réfrigérant qui assure d'une part l'arrêt de la réaction enzymatique, d'autre part une déprotéinisation de l'hydrolysat. Après refroidissement, les prélèvements sont centrifugés pendant $20 \mathrm{mn}$ à $4000 \mathrm{G}$. A $2 \mathrm{ml}$ du surnageant, on ajoute $\mathrm{I} \mathrm{ml} \mathrm{d'un} \mathrm{tampon} \mathrm{citraté} \mathrm{à} 20 \mathrm{p}$. roo (poids-volume) d'un mélange solide de citrate trisodique $(2,06 \mathrm{~g})$, d'acide citrique $(19,15 \mathrm{~g})$ et d'acide ascorbique $(0,5 \circ \mathrm{g})$ qui a pour effet d'amener le $\mathrm{pH}$ à $2,3-2,5$, pour procéder ensuite au dosage de l'azote aminé libéré.

Un témoin est réalisé simultanément en hydrolysant la protéine dans les mêmes conditions, sans la présence des prémélanoïdines.

\section{TABLEAU I}

Caractéristiques analytiques des prémélanoïdines de glucose et glycocolle étudiées

\begin{tabular}{|c|c|c|}
\hline & $\begin{array}{l}\text { Prémélanoïdines } \\
\quad\left(1 \mathrm{~h} 90^{\circ}\right)\end{array}$ & $\begin{array}{l}\text { Prémélanoïdines } \\
\qquad\left(6 \mathrm{~h} 90^{\circ}\right)\end{array}$ \\
\hline $\begin{array}{l}\text { Pouvoir réducteur total : } \\
\text { P. } 100 \text { du pouvoir réducteur initial ... }\end{array}$ & $8 i$ & 50 \\
\hline $\begin{array}{l}\text { Glucose résiduel : } \\
\text { P. } 100 \text { des prémélanoïdines totales } \ldots \ldots \\
\text { P. } 100 \text { du glucose initial........... }\end{array}$ & $\begin{array}{l}26,6 \\
37,8\end{array}$ & $\begin{array}{l}5,4 \\
7,7\end{array}$ \\
\hline $\begin{array}{l}\text { Glycocolle résiduel : } \\
\text { P. } 100 \text { des prémélanoïdines totales } \ldots \ldots \\
\text { P. } 100 \text { du glycocolle initial } \ldots \ldots \ldots \ldots\end{array}$ & $\begin{array}{l}14,2 \\
48,2\end{array}$ & $\begin{array}{r}5,3 \\
18,1\end{array}$ \\
\hline
\end{tabular}

De plus, comme les prémélanoïdines apportent dans le milieu d'hydrolyse une certaine quantité de glycocolle et d'autres substances réagissant à la ninhydrine dans le dosage de l'azote aminé, il est nécessaire de constituer un blanc. On a donc appliqué rigoureusement le processus expérimental décrit à une dose égale de prémélanoïdines, mais sans la présence des enzymes.

Les valeurs de dosages trouvées, exprimées en azote aminé, sont soustraites de la quantité d'azote aminé libéré par l'hydrolyse de la protéine en présence de prémélanoïdines.

Pour chaque protéine, les études suivantes ont été effectuées :

I. Hydrolyse enzymatique en présence de $500 \mathrm{mg}$ de prémélanoïdines " $6 \mathrm{~h}-90^{\circ}$ " par $\mathrm{g}$ de protéine.

II. Hydrolyse enzymatique en présence de $50 \mathrm{mg}$ de prémélanoïdines " $6 \mathrm{~h}-90^{\circ}$ " par $\mathrm{g}$ de protéine.

III. Hydrolyse enzymatique en présence de $100 \mathrm{mg}$ de prémélanoïdines " I h-900 " par $\mathrm{g} \mathrm{de}$ protéine ( $\left.{ }^{1}\right)$.

\section{E. - Dosage de l'azote aminé}

On procède au dosage de l'azote aminé total suivant une application de la méthode de Conway mise au point par MICHEL (I960). Par ébullition en milieu acide, la ninhydrine complexe l'ammoniac issu du groupement $\alpha$-aminé de l'acide aminé. On libère le $\mathrm{NH}_{3}$ par le perhydrol, et après déplacement par aération en milieu alcalin et microdiffusion dans une cellule de Conway, il est titré dans l'acide borique.

(1) Les doses de prémélanoïdines apparaissent comme arbitraires. En ce qui concerne les prémélanoïdines " I h-go , il ne nous a pas paru possible de reprendre les doses $50 \mathrm{mg}$ ou $500 \mathrm{mg}$ par g de protéine : compte tenu des quantités encore importantes de glucose et de glycocolle résiduels, $5^{\circ} \mathrm{mg}$ de prémélanoïdines totales nous paraissaient n'apporter en fait qu'une faible quantité de prémélanoïdines vraies.

Par contre, $500 \mathrm{mg}$ auraient apporté dans le blanc des valeurs d'azote aminé, dues au glycocolle résiduel, trop importantes. 


\section{RÉSULTATS}

Les résultats sont présentés dans les tableaux 2,3 et 4 .

I. Les doses faibles de prémélanoïdines « $6 \mathrm{~h}-90^{\circ}$ » (50 $\mathrm{mg} / \mathrm{g}$ de protéine) ne semblent pas apporter de modifications à la protéolyse des différents substrats (tabl. 2).

\section{TABLEAU 2}

Azote aminé libéré au cours de l'hydrolyse "pepsine-trypsine + peptidase" de différents substrats, en présence ou en absence de prémélanoidines " $6 h-90^{\circ}$ " (50 $\mathrm{mg} / \mathrm{g}$ de protéines)

(Chaque valeur est la moyenne de 2 à 4 déterminations)

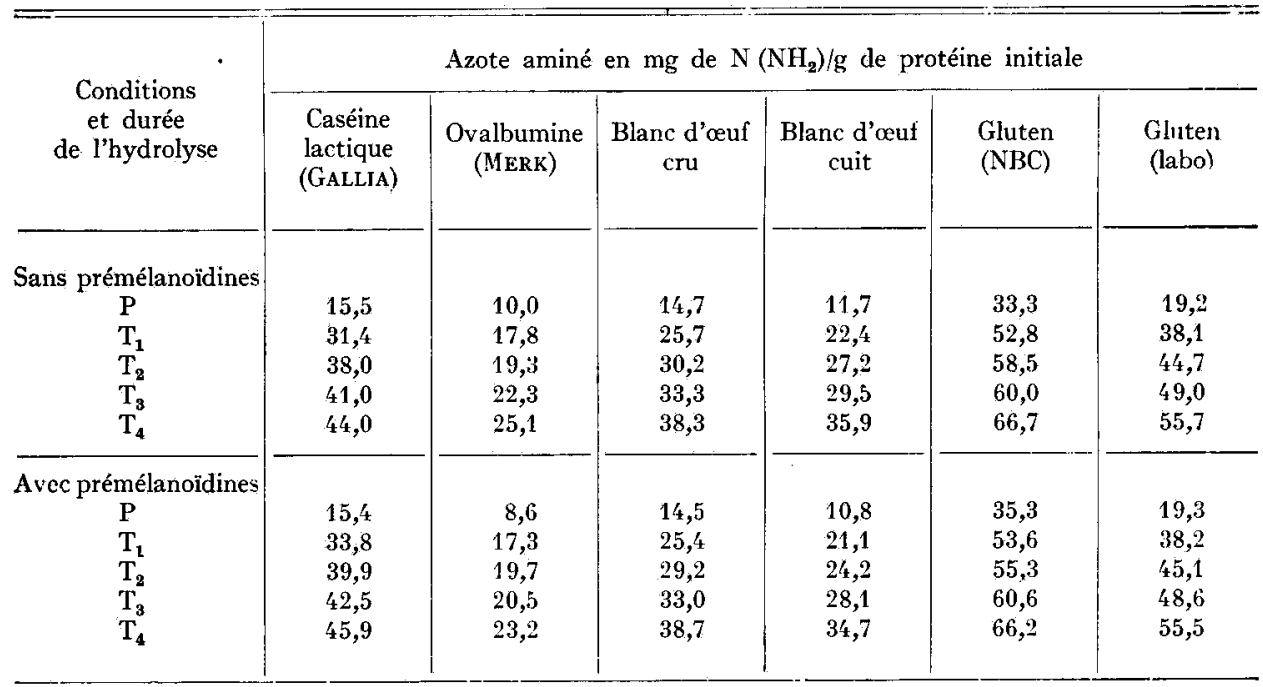

$\mathbf{P}=$ = prélèvement fin de l'hydrolyse pepsique $\mathrm{T}_{1}=$ prélèvement après $5 \mathrm{mn}$ d'hydrolyse trypsique $\mathrm{T}_{2}=$ prélèvement après $30 \mathrm{mn}$ d'hydrolyse trypsique
$\mathrm{T}_{3}=$ prélèvement après $1 \mathrm{~h}$ d'hydrolyse trypsique $\mathrm{T}_{4}=$ prélèvement après $3 \mathrm{~h}$ d'hydrolyse trypsique

2. Par contre, des différences apparaissent quand l'hydrolyse se déroule dans un milieu à forte concentration en prémélanoïdines (500 $\mathrm{mg} / \mathrm{g}$ de protéine) (tabl. 3 ) :

- la présence de prémélanoïdines se traduit, pour l'ovalbumine et les blancs d'œuf, par une quantité plus faible d'azote aminé libéré ;

- par contre, 1 hydrolyse du gluten et celle de la caséine ne semble pas affectées.

3. La présence de prémélanoïdines « I h- $90^{\circ}$ " à la dose de Ioo $\mathrm{mg} / \mathrm{g}$ de protéine semble n'avoir aucun effet sur la quantité d'azote aminé libéré au cours du temps (tab1. 4). 
TABLEAU 3. - Azote aminé liléré au cours de l'hydrolyse "pepsine-trypsine + peptidase" de différents substrats, en présence ou en absence de prémélanoïdines

" $6 h-90^{\circ}$ " (500 $\mathrm{mg} / \mathrm{g}$ de protéine)

(Chaque valeur est la moyenne de 2 à 4 déterminations)

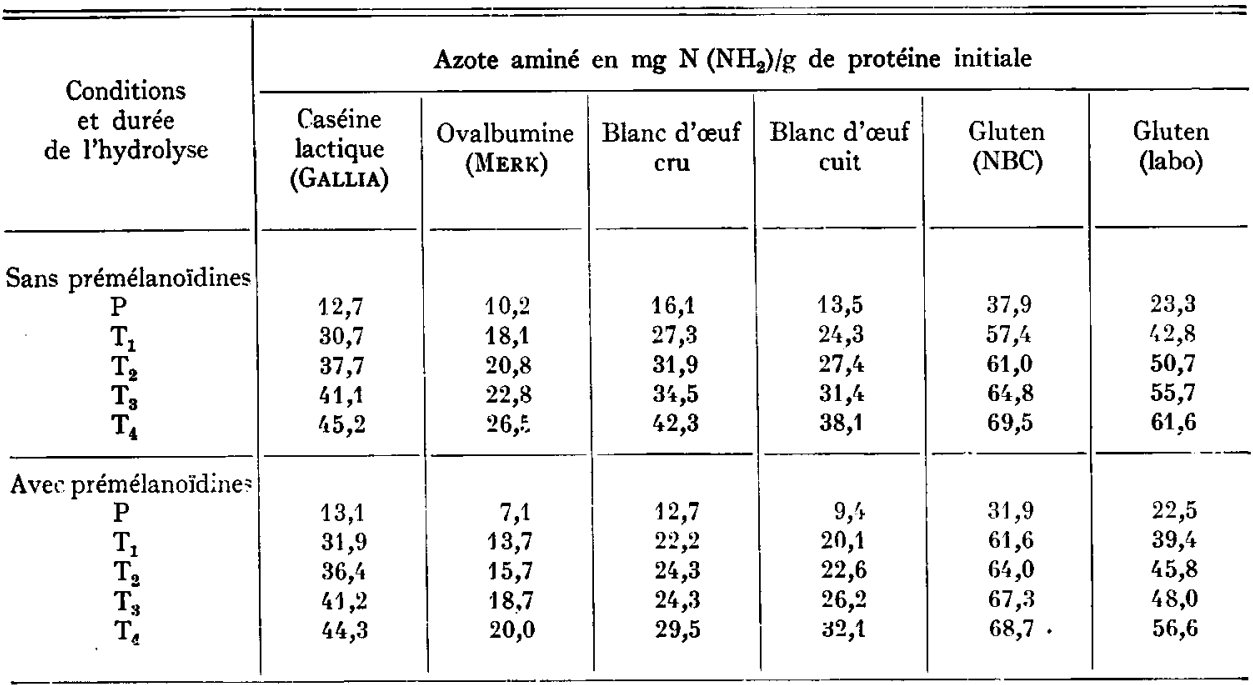

$\mathrm{P}=$ prélèvement fin de l'hydrolyse pepsique

$T_{1}=$ prélèvement après $5 \mathrm{mn}$ d'hydrolyse trypsique

$T_{2}=$ prélèvement après $30 \mathrm{mn}$ d'hydrolyse trypsique
$\mathrm{T}_{3}=$ prélèvement après $1 \mathrm{~h}$ d'hydrolyse trypsique $\mathrm{T}_{4}=$ prélèvement après $3 \mathrm{~h}$ d'hydrolyse trypsique

TABLEAU 4. - Azote aminé libéré au cours de l'hydrolyse "pepsine-trypsine + peptidase " de différents substrats en présence ou en absence de prémélanoïdines

" $1 \mathrm{~h}-90^{\circ}$ " (100 $\mathrm{mg} / \mathrm{g}$ de protéine)

(Chaque valeur est la moyenne de 2 à 4 déterminations)

\begin{tabular}{|c|c|c|c|c|c|c|}
\hline \multirow{2}{*}{$\begin{array}{l}\text { Conditions } \\
\text { et durée } \\
\text { de l'hydrolyse }\end{array}$} & \multicolumn{6}{|c|}{ Azote aminé libéré en $\mathrm{mg} \mathrm{N}\left(\mathrm{NH}_{2}\right) / g$ de protéine initiale } \\
\hline & $\begin{array}{l}\text { Caséine } \\
\text { lactique } \\
\text { (GALLIA) }\end{array}$ & $\begin{array}{c}\text { Ovalbumine } \\
\text { (MERK) }\end{array}$ & $\begin{array}{c}\text { Blanc d'œuf } \\
\text { cru }\end{array}$ & $\begin{array}{l}\text { Blanc d'œuf } \\
\text { cuit }\end{array}$ & $\begin{array}{l}\text { Gluten } \\
\text { (NBC) }\end{array}$ & $\begin{array}{c}\text { Gluten } \\
\text { (labo) }\end{array}$ \\
\hline $\begin{array}{c}\text { Sans prémélanoïdines } \\
\mathrm{P} \\
\mathrm{T}_{1} \\
\mathrm{~T}_{2} \\
\mathrm{~T}_{3} \\
\mathrm{~T}_{4}\end{array}$ & $\begin{array}{l}16,1 \\
34,8 \\
40,6 \\
42,3 \\
45,1\end{array}$ & $\begin{array}{l}10,8 \\
19,7 \\
22,5 \\
23,5 \\
27,3\end{array}$ & $\begin{array}{l}14,3 \\
25,3 \\
29,1 \\
33,6 \\
38,1\end{array}$ & $\begin{array}{l}12,6 \\
22,9 \\
26,2 \\
30,0 \\
37,3\end{array}$ & $\begin{array}{l}24,4 \\
41,0 \\
47,5 \\
48,6 \\
55,9\end{array}$ & $\begin{array}{l}18,3 \\
36, ; \\
43,6 \\
46,7 \\
54,8\end{array}$ \\
\hline $\begin{array}{c}\text { Avec prémélanoïdines } \\
\mathrm{P} \\
\mathrm{T}_{1} \\
\mathrm{~T}_{2} \\
\mathrm{~T}_{3} \\
\mathrm{~T}_{4}\end{array}$ & $\begin{array}{l}15,1 \\
32,6 \\
37,4 \\
41,8 \\
45,9\end{array}$ & $\begin{array}{l}10,2 \\
19,6 \\
22,5 \\
23,3 \\
27,4\end{array}$ & $\begin{array}{l}13,7 \\
23,1 \\
\because 7,1 \\
29,9 \\
36,0\end{array}$ & $\begin{array}{l}10,4 \\
20,2 \\
24,4 \\
27,3 \\
35,4\end{array}$ & $\begin{array}{l}26,3 \\
42,0 \\
49,7 \\
49,3 \\
53,3\end{array}$ & $\begin{array}{l}19,8 \\
36,8 \\
44,5 \\
46,5 \\
55,9\end{array}$ \\
\hline
\end{tabular}

$\mathbf{P}=$ prélèvement fin de l'hydrolyse pepsique

$T_{1}=$ prélèvement après $5 \mathrm{mn}$ d'hydrolyse trypsique

$\mathrm{T}_{2}=$ prélèvement après $30 \mathrm{mn}$ d'hydrolyse trypsique
$T_{3}=$ prélèvement après $1 \mathrm{~h}$ d'hydrolyse trypsique $\mathrm{T}_{\mathrm{t}}=$ prélèvement après $3 \mathrm{~h}$ d'hydrolyse trypsique 


\section{DISCUSSION}

I. Une première remarque s'impose à la lecture de ces tableaux : la cinétique de la protéolyse est sensiblement la même pour tous les substrats étudiés, mais, dans les conditions où nous étions placés, le gluten se distingue des autres par des quantités d'azote aminé libéré nettement plus élevées à tous les stades de 1'hydrolyse, en particulier dès la fin de 1'hydrolyse pepsique. Cette observation confirme celle de MouTONNET ( 1967$)$ : nous pensons donc comme lui qu'il serait intéressant de développer 1'étude de ce problème et de chercher quelle peut être sa portée pratique. Il est intéressant de rappeler à ce propos que, chez le Porc, l'élévation de l'aminoacidémie porte se produit rapidement après ingestion d'une ration à base de blé (Pron et RÉRAT, I967).

D'autre part, on voit que, dans le cas du gluten N. B. C., l'hydrolyse ne s'est pas déroulée identiquement au cours des trois séries d'essais : elle est plus lente pour celle qui est rapportée dans le tableau 4 que pour les deux autres. Tous ces essais ont porté cependant sur le même échantillon, mais un délai de six mois s'est écoulé entre les deux premières séries et la troisième. Il ne nous est malheureusement pas possible de dire si cette différence est due à une évolution de l'échantillon de gluten ou à un changement dans les échantillons d'enzymes employés.

Il n'en demeure pas moins que les comparaisons entre l'hydrolyse en présence ou en l'absence de prémélanoïdines sont légitimes, puisque, à l'intérieur d'une même série, les essais ont été effectués simultanément.

2. Quant au rôle des prémélanoïdines, on ne saurait, sur ces seuls résultats, expliquer la différence de comportement des protéines étudiées en présence d'une forte dose de prémélanoïdines. On remarque que seules sont affectées les protéines de l'œuf, et ceci dès la fin de l'hydrolyse pepsique. Il est donc logique d'en déduire que la cause du retard de la libération de l'azote aminé en présence de prémélanoïdines est à rechercher dans les caractéristiques propres des protéines de l'œuf. Ce problème pourra justifier lui aussi de nouvelles recherches.

3. Du point de vue pratique, aucun de nos résultats ne conduit à penser que les produits solubles de la réaction de Maillard puissent entraver l'attaque des protéines par les enzymes protéolytiques. Dans le seul cas où nous avons observé un ralentissement de la protéolyse, les doses de prémélanoïdines étaient considérables.

Rę̧u pour publication en février 1969.

\section{REMERCIEMENTS}

Nous adressons nos vifs remerciements à MM. GuILBot et PeTIT pour leur accueil dans le Laboratoire de Biochimie et Physicochimie des Céréales (INRA, Massy) où une partie de ce travail a été effectuée. 


\section{SUMMARY}

\section{INFLUENCE OF SOLUBLE PRODUCTS FORMED BY HEATING GLUCOSE AND GLYCOCOL ON ENZYMIC HYDROLYSIS OF DIFFERENT PROTEINS}

By heating glucose and glycocol at $90^{\circ} \mathrm{C}$ for I or 6 hours a mixture of soluble products, premelanoidins, was obtained. Their influence on hydrolysis in vitro of some proteins was studied. The proteins were casein, ovalbumin, raw or cooked egg white and wheat gluten. The enzymes used were pepsin, trypsin and peptidase.

At a concentration of $100 \mathrm{mg}$ per g protein the pre-melanoidins obtained after I hour at $90^{\circ} \mathrm{C}$ did not affect hydrolysis of the different proteins studied. At $50 \mathrm{mg}$ per g protein the pre-melanoidins obtained after 6 hours at $90^{\circ} \mathrm{C}$ also did not affect hydrolysis of the differrent proteins studied. At $50 \mathrm{mg}$ per $\mathrm{g}$ protein, the pre-melanoidins obtained after 6 hours at $90{ }^{\circ} \mathrm{C}$ also did not affect hydrolysis of the proteins. At a concentration ten times as great, $500 \mathrm{mg}$, they reduced the amount of nitrogen liberated from albumin or egg white, but did not modify proteolysis of the other substrates. The proteins of egg thus had a specific mode of behaviour, probably because of their physical properties.

\section{RÉFÉRENCES BIBLIOGRAPHIQUES}

Adrian J., Frange R., Petit L., Godon B., Barbier J., 1966. Répercussions nutritionnelles des produit solubles formés au cours de la réaction de Maillard. Ann. Nutrit. Alim., 20, 257-277.

GuidotTi G., CоLомвo J. P., FoA P. P., I96I. Enzymatic determination of glucose : stabilisation of color developped by oxidation of orthodianisidine. Anal. Chem., 83, $151^{-1} 53$.

Huggett A., Nixon D. A., I957. Enzymatic determination of blood glucose. Bioch. J., 68, I2 Proc.

JemmaLI M., 1965. Sur le comportement de deux lypes de microorganismes et de différents enzymes en présence de produits de la réaction de Maillard. Thèse de Docteur-Ingénieur, Fac. Sci., Paris, no ordre 942, 6 mai 1965.

Micher M. C., 1960. Technique de microdosage de l'azote $\alpha$-aminé : dosage de l'azote aminé dans quelques liquides biologiques. Amino-acides, pepiides, proteines, cahier n 4, 63-71. A. E. C., Commentry.

Moutonnet P., 1967. Influence des sulfites sur la protéolyse : étude in vitro. Ann. Biol. anim. Bioch. Biophys., 7, 63-7I.

PetiT L., AdRIAN J., 1967. La réaction de Maillard. Description et répercussions physiologiques. Cahiers Nutrit. Diet., 2, $3 \mathrm{I}-38$.

Pion R., RÉrat A., 1967. Influence d'une supplémentation en lysine sur l'évolution de l'aminoacidémie porte du porc en croissance, au cours de la digestion d'une ration à base de blé. C. R. Acad. Sci., 264, D, $632-635$.

Schaffer P. A., Hartmann A. F., 1920. The iodometric determination of copper and its use in sugar analysis. II. Methods for determination of reducing sugars in blood, urine, milk and other solutions. $J$. biol. Chem., 45, 365-390.

Sheik N. M., Godon B., Petrt L., 196i. Influence des produits de la réaction de Maillard sur la fermentation alcoolique et le développement de la levure. Ann. Technol. agric., 10, 5-42.

Staron T., Altard C., Chambre M. M., 196r. Nouvelle méthode de chromatographie sur papier et de dosage des acides aminés. C. R. Acad. Sci., 253, 1630-1632. 